\title{
Cross-fit T cells battle Zika virus
}

Flaviviruses stimulate cross-reactive immune responses that may reduce or exacerbate manifestations of subsequent flavivirus infection. Recent work demonstrates that cross-reactive T cells protect against Zika in HLA transgenic mice, a key step in the development of safe and effective vaccines.

\section{Matthew Collins and Aravinda de Silva}

ika virus infection was declared a public health emergency by the World Health Organization in 2016 because of its rapidly expanding incidence and association with novel phenotypes, including sexual transmission and birth defects. As Zika and the closely related dengue viruses share the same mosquito vector, these viruses co-circulate in the same communities. Flaviviruses stimulate strongly cross-reactive immune responses in humans. Views of cross-reactive (heterologous) immunity in the flavivirus field are strongly coloured by the wellsupported hypothesis of antibodydependent enhancement, whereby nonneutralizing, cross-reactive antibodies elicited by previous infection with a a

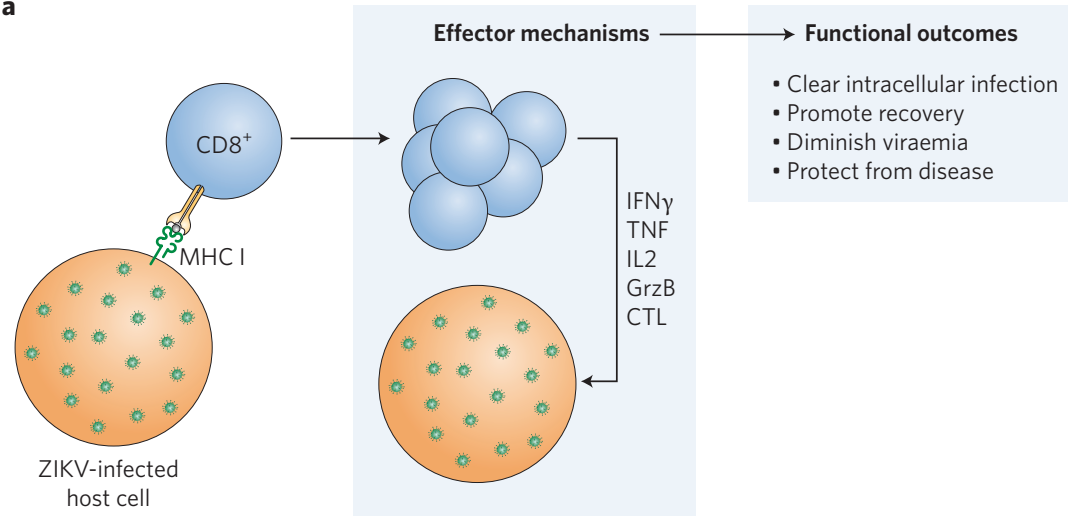

heterologous dengue serotype increase infectivity of Fc-receptor-bearing cells, leading to higher viraemia and greater risk for severe disease ${ }^{1}$. Although Zika virus is closely related to dengue, the extent and effects of immunologic cross-reactivity between Zika and dengue are unknown ${ }^{2}$.

Two recent publications ${ }^{3,4}$ from Sujan Shresta's group at the La Jolla

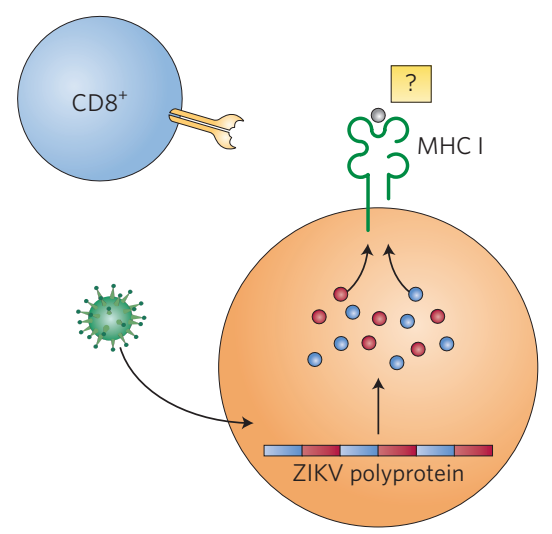

c ZIKV infection in DENV-immune host

$\mathrm{CD} 8^{+} \mathrm{T}$ cell population
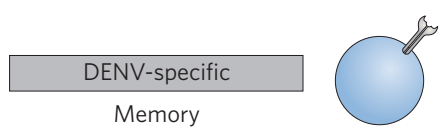

ZIKV-specific

Naive repertoire

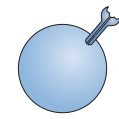

DENV-ZIKV cross-reactive

Cross-reactive memory

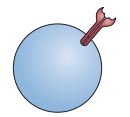

Effect of ZIKV exposure

No effect

Priming and generation of effector and memory response with primary kinetics

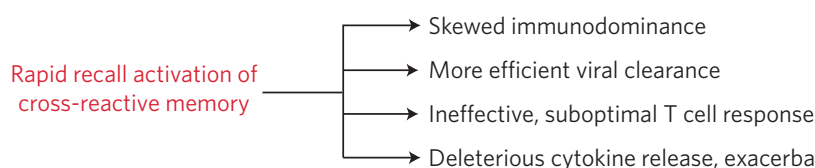

Deleterious cytokine release, exacerbated pathology

Figure 1 l CD8 ${ }^{+} T$ cell responses to Zika virus infection. $\mathbf{a}$, Zika virus infection or vaccination elicits $C D 8^{+} T$ cells that are protective in some animal models. b. The epitopes targeted by Zika-reactive $C D 8^{+} T$ cells in humans are being identified. Zika-derived peptides presented by HLA can either be unique to Zika or conserved between Zika and closely related flaviviruses (blue represents peptide epitopes unique to Zika virus and red those conserved between Zika virus and dengue). c, How will existing dengue immunity affect $T$ cell responses upon Zika infection? Dengue-virus-specific memory $C D 8^{+} T$ cells are unlikely to be activated by Zika exposure. Epitopes unique to Zika virus will prime Zika-specific naïve CD8 ${ }^{+} \mathrm{T}$ cells. Pre-existing cross-reactive memory $T$ cells may be protective, ineffective, or disease enhancing. Wen et al. demonstrate a protective role for cross-reactive CD8 ${ }^{+} \mathrm{T}$ cells in HLA transgenic mice. ZIKV, Zika virus; DENV, dengue virus; IFN, interferon; TNF, tumour necrosis factor; IL, interleukin; GrzB, granzyme B, CTL, cytotoxic T lymphocyte. 
Institute of Allergy and Immunology, one of which appears in this issue of Nature Microbiology, use innovative mouse models to dissect the $\mathrm{CD} 8^{+} \mathrm{T}$ cell response to Zika virus (Fig. 1). CD8 ${ }^{+} \mathrm{T}$ cells mediate immunity to intracellular infections by recognizing and responding to specific peptides that are derived from intracellular proteins and presented on class I major histocompatibility complex (MHC) molecules. The role of $\mathrm{CD}^{+} \mathrm{T}$ cells in dengue pathogenesis is unclear: certain class I MHC haplotypes have been associated with increased or decreased rates of severe dengue $^{5}$; however, these studies do not consistently include analysis of $\mathrm{T}$ cell function, along with human leukocyte antigen (HLA) typing. Earlier work suggested that cross-reactive $\mathrm{T}$ cell responses from prior dengue infection precipitate a dysfunctional profile of cytokine secretion and cytolysis by activated T cells ${ }^{6}$, but growing data indicate that individuals with more robust $\mathrm{T}$ cell immunity are less likely to develop symptoms or severe manifestations of homologous or heterologous dengue infection ${ }^{5}$.

As the mouse type I interferon response blocks the replication of $\mathrm{Zika}$, Elong Ngono et al. used mice lacking the type I interferon receptor in a subset of myeloid cells as a model to study CD8 ${ }^{+}$ $\mathrm{T}$ cell immunity to $\mathrm{Zika}^{4}$. In this model, the virus replicated and induced a robust $\mathrm{CD}^{+} \mathrm{T}$ cell response. Using transgenic mice lacking $\mathrm{CD}^{+} \mathrm{T}$ cells or passive transfer of $\mathrm{CD}^{+} \mathrm{T}$ cells from Zika-immune to naïve mice, $\mathrm{CD} 8^{+} \mathrm{T}$ cells were shown to reduce viral replication and ameliorate disease. These observations are similar to previous reports demonstrating that $\mathrm{CD}^{+} \mathrm{T}$ cells are protective in mouse models of dengue infection ${ }^{7,8}$. Collectively, these studies demonstrate that, in addition to the wellestablished role of antibodies in protective immunity, $\mathrm{CD}^{+} \mathrm{T}$ cells also contribute to protective immunity against flaviviruses. A limitation of these studies is that the experiments must be carried out in mice with substantially altered immune systems in order to achieve productive flavivirus infection. Moreover, the specific viral epitopes targeted in mice are unlikely to be the same as in humans.
In this issue of Nature Microbiology, Wen et al. use type I interferon receptordeficient mice expressing human HLA proteins (HLA-B ${ }^{\star} 0702$ and HLA-A*0101) to identify Zika peptides that are likely to be targeted by human $\mathrm{CD} 8^{+} \mathrm{T}$ cells. Given the recent emergence of severe Zika cases in areas with endemic dengue transmission, many groups are now exploring whether dengue immunity modifies Zika disease or vice versa. Wen and colleagues also use the HLA transgenic mouse model to explore the role of prior dengue infection in shaping the $\mathrm{T}$ cell response to Zika infection.

What have we learned from Wen et al.? First, their data agree with other recent work in animal models of infection and vaccination showing that Zika virus elicits antigen-specific $\mathrm{CD}^{+} \mathrm{T}$ cell responses ${ }^{4,9-11}$. These $\mathrm{T}$ cell responses clearly have a protective role in the Shresta mouse model $^{4}$, although they were not required for suppressing viraemia in a Balb/c mouse model of Zika vaccination ${ }^{11}$. Second, the HLA transgenic mouse model has previously demonstrated utility in identifying relevant epitopes targeted by human $\mathrm{CD} 8^{+} \mathrm{T}$ cell responses across a number of HLA alleles and viruses ${ }^{12}$. It is important to note that Wen et al. identified distinct epitopes compared to the $\mathrm{Bl} / 6$ mouse model ${ }^{4,9}$, further indicating that there are differences in determinants of immunodominance between species.

Wen et al. show that HLA transgenic mice infected with Zika or dengue develop $\mathrm{CD}^{+} \mathrm{T}$ cells that cross-react with several peptides that are conserved between Zika and dengue virus. They also demonstrate that dengue and Zika virus cross-reactive $\mathrm{CD}^{+} \mathrm{T}$ cell responses can be protective against Zika; however, this is not definitive proof that dengue infection will be protective against subsequent Zika infection in humans (Fig. 1c). Wen et al. used a computational method to predict the top class I MHC epitopes encoded by the Zika genome and tested these peptides for cross-reactivity. A more comprehensive (though less practical) approach to address this question would require screening for reactivity against all possible Zikaderived peptide epitopes following dengue immunity. In fact, it may be that weakly cross-reactive $\mathrm{T}$ cell responses to Zika are the most problematic ${ }^{6}$, particularly if these come at the expense of more robust $\mathrm{CD}^{+}$ $\mathrm{T}$ cell effector function or $\mathrm{CD} 4^{+} \mathrm{T}$ cell help for developing strongly neutralizing antibody responses.

Many unknowns remain, but through the identification of epitopes that are likely to be targeted by the human $\mathrm{CD}^{+} \mathrm{T}$ cell response to Zika, Wen et al. illuminate the way forward. Both Wen et al. and Elong Ngono et al. contribute to mounting evidence that $\mathrm{T}$ cells are critical players in immunity to flaviviruses and should be considered for vaccine development. Several dengue vaccines under development or in clinical trials do not include native antigens known to encode dominant T cell epitopes, and such approaches may need revisiting in view of the work discussed here. Validation of Zika epitopes recognized by $\mathrm{CD}^{+} \mathrm{T}$ cells from exposed human subjects is likely to follow soon. The HLA transgenic mouse system also provides an important tool for identification of $\mathrm{CD}^{+} \mathrm{T}$ cell epitopes that could quickly be translated into MHC class I tetramer reagents for tracking antigen-specific responses in humans and for performing mechanistic studies in this experimental model.

Matthew Collins is with the Division of Infectious Diseases in the Department of Medicine, University of North Carolina School of Medicine, Chapel Hill, North Carolina 27599, USA. Aravinda de Silva is in the Department of Microbiology and Immunology, University of North Carolina School of Medicine, Chapel Hill, North Carolina 27599, USA. e-mail:aravinda_desilva@med.unc.edu

\footnotetext{
References

1. Halstead, S. B. F1000Res. http://doi.org/b6m9 (2015).

2. Lazear, H. M. \& Diamond, M. S. J. Virol. 90, 4864-4875 (2016).

3. Wen, J. et al. Nat. Microbiol. 2, 17036 (2017).

4. Elong Ngono, A. et al. Cell Host Microbe 21, 35-46 (2017).

5. Weiskopf, D. \& Sette, A. Front. Immunol. 5, 93 (2014).

6. Rothman, A. L. J. Clin. Invest. 113, 946-951 (2014).

7. Zellweger, R. M. J. Virol. 89, 6494-6505 (2015).

8. Yauch, L. E. et al. J. Immunol. 182, 4865-4873 (2009).

9. Pardy, R. D. et al. PLoS Pathog. 13, el006184 (2017).

10. Abbink, P. et al. Science 353, 1129-1132 (2016).

11. Larocca, R. A. et al. Nature 536, 474-478 (2016).

12. Weiskopf, D. et al. J. Immunol. 187, 4268-4279 (2011).
}

Competing interests

A.d.S. has consulted on dengue vaccines for Takeda

Vaccines, Glaxo Smith Kline and Merck Pharmaceuticals.

A.d.S. is an inventor on patents related to dengue vaccines. 